\title{
Challenges to improve antenatal and intrapartum care in South Africa
}

\author{
R C Pattinson, MD, FRCOG, FCOG (SA); T M A G Hlongwane, MB ChB, FCOG (SA) MMed; V Vannevel, MD, MMed (O\&G) \\ Department of Obstetrics and Gynaecology, School of Medicine, Faculty of Health Sciences, and Research Centre for Maternal, Fetal, Newborn and \\ Child Health Care Strategies, University of Pretoria, and Maternal and Infant Health Care Strategies Research Unit, South African Medical Research \\ Council, Pretoria, South Africa
}

Corresponding author: V Vannevel (valerie.vannevel@up.ac.za)

\begin{abstract}
The major causes of maternal and perinatal deaths have been well described in South Africa. These causes are related to HIV infection, placental insufficiency and intrapartum asphyxia. The health system failures that most commonly lead to preventable mortality are related to managing hypertensive disorders in pregnancy (HDP), detecting fetal growth restriction antenatally and managing labour effectively by providing caesarean delivery to those who need it and avoiding it in those who do not. Improving antenatal and intrapartum care are vital aspects in efforts to improve survival, but to achieve this the following challenges need to be overcome:

- managing the increased antenatal care contacts needed to detect HDP

- creating a next level of expertise, and access for women to high-risk care

- creating the environment for respectful care and companionship in labour

- managing labour as physiologically as possible

- detecting and managing placental insufficiency.

This article provides some exciting solutions to these health system barriers.
\end{abstract}

S Afr Med J 2019;109(11 Suppl 1):S15-S19. https://doi.org/10.7196/SAMJ.2019.v109i1 1b.14248

South Africa (SA) has committed itself to achieving the Sustainable Development Goals (SDGs). Goal 3 is to ensure healthy lives and promote wellbeing for all, at all ages. For maternal and neonatal health specifically, the targets are to: (i) reduce the global maternal mortality ratio (MMR) to <70 per 100000 live births; and (ii) end preventable deaths of newborns and children under 5 years of age - with all countries aiming to reduce neonatal mortality (deaths during the first 28 days of life) to at least as low as 12 per 1000 live births, and under-5 mortality to at least as low as 25 per 1000 live births by $2030 .{ }^{[1]}$ In 2015, StatsSA reported our baseline MMR as 118 per 100000 live births, while the neonatal mortality rate was 12 per 1000 live births. ${ }^{[2]}$ Therefore, we have some way to go in reducing our MMR, but the neonatal death rate goal has been achieved.

This, however, is only part of the story, as our stillbirth rate (not included in the SDGs) is more than three times that of neonatal deaths for babies weighing $\geq 1000$ g. ${ }^{[3]}$ From 2015 to 2017, there were 2558397 births recorded in the Perinatal Problem Identification Programme (PPIP), constituting $82 \%$ of all births that occurred in the public sector. Of these births recorded in PPIP, 45591 were perinatal deaths: 20757 (45.5\%) were antenatal stillbirths; 10609 (14.2\%) were intrapartum deaths and 14225 (31.2\%) were early neonatal deaths. ${ }^{[3]}$ The Saving Babies reports collate the most common underlying causes/categories of perinatal deaths: unexplained stillbirths; hypertensive disorders in pregnancy (HDP); antepartum haemorrhage (APH); intrapartum asphyxia and birth trauma (IPA\&T); and spontaneous preterm birth (SPTB). ${ }^{[4]}$ The Saving Mothers reports give the major underlying causes of maternal death: non-pregnancyrelated infections (NPRI) ( 95\% HIV-infected); HDP; obstetric haemorrhage $(\mathrm{OH})$; and pre-existing medical and surgical conditions (M\&S). ${ }^{[5]}$ Analysis of these causes indicates that the areas where the health system needs to concentrate to prevent these conditions are in antenatal care (NPRI, HDP, APH, pre-existing M\&S and unexplained stillbirths) and labour (postpartum haemorrhage (PPH), IPA\&T and SPTB). ${ }^{[5]}$

The major complicating factors that lead to these deaths are HIV infection, placental insufficiency and complicated labour often associated with caesarean delivery (CD). HIV infection results in increased comorbidity, such as $\mathrm{TB}$, meningitis, pneumonia and preterm delivery. Placental insufficiency is the underlying cause of complications such as HDP, abruptio placentae and a significant proportion of unexplained stillbirths. Complicated labour results in increased CDs and postpartum haemorrhage, as well as IPA\&T. ${ }^{[4,5]}$ Although improving antenatal and intrapartum care are important in reducing mortality, other aspects such as preventing deaths from $\mathrm{OH}$ are also extremely important.

The World Health Organization (WHO) has recently published two major sets of recommendations: 'The WHO recommendations for a positive pregnancy experience' (November 2016), ${ }^{[6]}$ relating to antenatal care (ANC), and 'The WHO recommendations for a positive childbirth experience' (January 2018), ${ }^{[7]}$ relating to intrapartum care (IPC). Both these sets of recommendations have major implications for the provision of ANC and IPC, and also address in part the major causes of maternal and newborn mortality. In both cases, task teams in SA have been set up to analyse the implications and to consider adaptations to SA guidelines to help reduce our mortality rates. The SA Medial Research Council played an integral role in adapting the guidelines to meet our needs and to bring in the thrive-andtransform aspect of care, and not only the survive aspect.

\section{Antenatal care}

Basic ANC (BANC) was introduced in SA in 2008, and followed the WHO model, with 4 goal-orientated contacts. ${ }^{[8]}$ An audit of the 
timing and causes of all stillbirths in three provinces in SA showed that HDP and unexplained stillbirths were the most common causes of adverse perinatal outcomes, and that the third trimester was identified as a crucial time, with a peak in stillbirths between 32 and 38 weeks. ${ }^{[9]}$ With the BANC model, stillbirths were not being prevented, but diagnosed. Based on local findings and global evidence, the SA National Department of Health (NDoH) adopted the Basic Antenatal Care Plus (BANC Plus) guideline for antenatal care, and at-scale implementation started in April 2017. This entailed, among other changes, increasing the number of contacts to a minimum of eight, mostly concentrated in the third trimester. ${ }^{[6]}$

Evaluation of implementation of BANC Plus occurred at 4 sites across SA. All 4 catchment areas had BANC Plus training before April 2017. The antenatal record and pregnancy outcomes were recorded in 50 consecutive deliveries before implementation of BANC Plus (March 2017) and subsequently for each quarter (July, September, November 2017 and February 2018), resulting in 1000 records to analyse. Data on perinatal mortality were collated through the PPIP, and analysed in 6-monthly periods to assess any impact on mortality: the 6 months before the change in antenatal care guidelines (October 2016 - March 2017, i.e. control group); the first 6 months into the change (April 2017 - September 2017, i.e. during transition); and in the following 6 months (October 2017 - March 2018, i.e. after transition). ${ }^{[10]}$
Over time the number of antenatal care contacts increased; mean contacts being 4.85 in March 2017 to 5.96 in February 2018. In March 2017, 4.6\% and 36.5\% of women had 8 and 6 contacts or more, respectively; in February 2018, this had increased to $24.5 \%$ and $61 \%$, respectively. There was an improvement in measurement of blood pressure (BP), testing for proteinuria and diagnosing hypertension in pregnancy: a 30\% increase in hypertension detection from the baseline to February 2018, as well as a $23 \%$ increase in the number of cases where appropriate action was taken. However, one-third of women were still not appropriately managed one year after the implementation of BANC Plus. Furthermore, evaluation of the perinatal deaths revealed no change in perinatal mortality, but a substantial reduction in unexplained stillbirths (from 10.7 to 5.6/1 000 Tables 2 and 3) and a substantial increase in stillbirths where hypertension was given as the underlying cause (1.5 to 3.1/1 000 Tables 2 and 3). ${ }^{[10]}$ The most common avoidable factor was 'not responding to antenatal hypertension' (Table 4) and this correlates with the number of cases where appropriate action for managing hypertension was not taken during ANC. ${ }^{[3]}$

Thus, the core problem in perinatal deaths due to HDP lies with the primary healthcare clinics ( $\mathrm{PHC}$ ) not managing the woman appropriately by either not referring her to a higher level facility or having a more skilled attendant manage the pregnancy. Lack of recognition of the problem and full assessment at the primary

Table 1. Key targets to ensure MNC patients survive, thrive and transform ${ }^{[1]}$

\section{Survive}

- End preventable deaths

- Reduce global maternal mortality to less than 70 per 100000 live births

- Reduce newborn mortality to at least as low as 12 per 1000 live births in every country

- Reduce under-5 mortality to at least as low as 25 per 1000 live births in every country

- End epidemics of HIV, tuberculosis, malaria, neglected tropical diseases and other communicable diseases

- Reduce by one third premature mortality from non-communicable diseases and promote mental health and well-being

Thrive

- Ensure health and well-being

- End all forms of malnutrition and address the nutritional needs of children, adolescent girls, and pregnant and lactating women

- Ensure universal access to sexual and reproductive healthcare services (including family planning) and rights

- Ensure that all girls and boys have access to good-quality early childhood development

- Substantially reduce pollution-related deaths and illnesses

- Achieve universal health coverage, including financial risk protection and access to quality essential services, medicines and vaccines

Transform

- Expand enabling environments

- Eradicate extreme poverty

- Ensure that all girls and boys complete free, equitable and good-quality primary and secondary education

- Eliminate all harmful practices and all discrimination and violence against women and girls

- Achieve universal and equitable access to safe and affordable drinking water and to adequate and equitable sanitation and hygiene

- Enhance scientific research, upgrade technological capabilities and encourage innovation

- Provide a legal identity for all, including birth registration

- Enhance the global partnership for sustainable development

$\mathrm{MNC}=$ maternal, neonatal and child

Table 2. PPIP indicators: Primary causes of Stillbirths ${ }^{[10]}$

\begin{tabular}{lllllll}
\hline Time period & Total del. & Del. ${ }^{*}$ & Unexp. SB & HDP & APH & SpPD \\
\hline Oct 2016 - Mar 2017 & 9801 & $9637^{*}$ & 103 & 14 & 39 & 11 \\
Apr 2017 - Sep 2017 & 10581 & $10408^{*}$ & 65 & 39 & 28 & 18 \\
Oct 2017 - Mar 2018 & 11051 & $10866^{*}$ & 61 & 34 & 40 & 19
\end{tabular}

PPIP = October 2016 - March 2018; HDP = hypertensive disorders in pregnancy; del. = 31 433; Unexp. = unexplained; APH = antepartum haemorrhage; SpPD = spontaneous preterm delivery. 
Table 3. Stillbirth rates due to hypertension, unexplained stillbirths and antepartum haemorrhage in antenatal care attenders

\begin{tabular}{llll}
\hline Time period & HPD SBR & Unexplained SBR & APH SBR \\
\hline Oct 2016 - Mar 2017 & 1.5 & 10.7 & 2.5 \\
Apr 2017 - Sep 2017 & 3.7 & 6.2 & 2.7 \\
Oct 2017 - Mar 2018 & 3.1 & 5.6 & 3.6 \\
HPD = hypertensive disorders in pregnancy; SBR = stillbirth rate; APH = antepartum haemorrhage. & & \\
${ }^{*}$ Births and stillbirths $>$ 1 000 g. & &
\end{tabular}

Table 4. Primary cause of death and avoidable factors

\begin{tabular}{|c|c|c|c|c|}
\hline \multirow[b]{2}{*}{ Time period } & \multicolumn{2}{|c|}{ Stillbirths } & \multicolumn{2}{|c|}{ Stillbirths and early neonatal deaths } \\
\hline & HDP, $n$ & No response, $n(\%)$ & HDP, $n$ & No response, $n$ (\%) \\
\hline Oct 2016 - March 2017 & 14 & $9(64)$ & 19 & $9(47)$ \\
\hline April 2017 - Sep 2017 & 39 & $22(56)$ & 48 & $27(56)$ \\
\hline Oct 2017 - March 2018 & 34 & $15(44)$ & 38 & $18(47)$ \\
\hline
\end{tabular}

care level was also the most common avoidable factor by a health care professional, for women who died due to complications of hypertension in pregnancy. ${ }^{[3,5]}$

In SA, deaths due to HDP are the most common direct cause of maternal mortality and account for $14.8 \%$ of all maternal deaths. ${ }^{[5]}$ HDP deaths have increased in importance as potentially preventable deaths as management of the two other major conditions, nonpregnancy related infections and obstetric haemorrhage, has improved. No progress has been made in the last decade in reducing the maternal deaths due to HDP. Most of the HDP deaths were thought to be potentially preventable, and this percentage has increased over the last few years and is now assessed to be at $\sim 75 \%$ in 2017. ${ }^{[5]}$ Timely detection and appropriate management will reduce the percentage of preventable deaths.

The Minister of Health and the National Health Council have decided to prioritise HDP in 2019. The new management of HDP guidelines were reviewed and rewritten to ensure that they are comprehensive and deal with all levels of care. ${ }^{[11]}$ The guidelines and implementation plan have been accepted by the NDoH. To be successful, women with HDP must be detected (through increased contacts) and managed at the appropriate level of care (not done currently). The guidelines introduce the concept of 'next level of expertise' (NLE), an innovation that promotes creating a functional NLE at the PHC or community health centre and make expert care accessible to all women. There are several ways in which this can be achieved, where an advanced antenatal care practitioner (doctor or advanced midwife) is able to deal with women with moderate risks at the clinic without her having to travel to another site/level of care.

Focus groups around the country revealed that although there were options to deal with women with hypertension, not many districts had specific plans. Targeting PHCs that perform ANC, improving their knowledge and ensuring a functioning referral system to the NLE are essential to reduce maternal and perinatal deaths due HPD.

There are five models used to manage the increased load of highrisk pregnant women:

- training an 'advanced antenatal care practitioner' (advanced midwife or doctor) to see the cold referrals weekly at the clinic

- training the doctors at the PHC to assess the cold referrals and decide on referral to the hospital or specialist. A training course (BANC Plus consultation) was developed for this

- outreach from the hospital by doctors from the maternity unit to see women at the $\mathrm{PHC}$
- outreach from the District Clinical Specialist Team (DCST) to the $\mathrm{PHC}$

- use of video conferencing with the midwife and doctor through a smart phone application.

The solutions will vary across different catchment areas based on the local geography and unique circumstances. Until the barrier of access to quality ANC is resolved, reduction in deaths due to HDP is unlikely.

\section{Intrapartum care}

The monitoring of women in labour, using the partogram and an active management, has remained unchanged for a long time. However, in view of the increased number of interventions during labour there has been a renewed interest in the natural progression of labour. One question could be posed: in an attempt to align all women to the same norm, are we not causing harm?

As a result of the studies published by Zhang et al. ${ }^{[12,13]}$, the WHO group has done more research on labour progression. A systematic review on the cervical dilatation patterns in low-risk labouring women with normal perinatal outcomes was published, describing a more rapid progress of labour from $6 \mathrm{~cm}$ onwards. However, in some women, labour progression is slower than $1 \mathrm{~cm} /$ hour, even in advanced labour. These findings led to the conclusion that the 1 $\mathrm{cm}$ /hour per hour rule may be unrealistically fast for some healthy women, who will still give birth to a healthy baby vaginally. ${ }^{[14]}$

The BOLD project, led by WHO, was a prospective, multi-centre cohort study which aimed to develop a new labour monitoring-toaction tool, done in Nigeria and Uganda. In one paper, they examined the labour progression of women with a spontaneous onset of labour and subsequent vaginal birth with normal perinatal outcome. Results showed that the slowest-yet-normal rate to progress can be slower than the $1 \mathrm{~cm} /$ hour norm. ${ }^{[15]}$

The predictive value of the cervical dilatation for adverse birth outcomes is described in another paper: of the 9995 women included, $2.2 \%$ had severe adverse birth outcomes but $49 \%$ of women had crossed the alert line. ${ }^{[16]}$ Cervical dilatation had a poor performance as a predictor of severe birth outcomes which led to the main conclusion that the validity of the partogram with the alert and action lines should be re-evaluated.

Based on this research, the WHO published the new recommendations on Intrapartum Care for a Positive Childbirth 
Experience. ${ }^{[7]}$ The recommendations discourage interventions during labour solely based on progress of cervical dilatation: if the mother's and the baby's condition is reassuring, there is no need to intervene for slow progress alone.

To our knowledge, SA has been a pioneer in implementing the new WHO recommendations into its IPC guideline. The process of updating the guideline was extensive and involved all stakeholders. Two aspects stand out: (i) focus on respectful, woman-centred care: often neglected in favour of the 'medical' aspects of labour but known to improve maternal and neonatal outcome; and (ii) changes in the monitoring of labour: duration of latent phase allowed up to 24 hours, start of active phase at $5 \mathrm{~cm}$ and less vaginal examinations.

As a first step towards implementing the new guideline, SA has updated the partogram in the Maternity Case Record to an 'interim' partogram while awaiting a new WHO labour monitoring tool.

Many possible issues have been raised during development of the guideline:

- Will longer labour lead to more babies with IPA?

- When looking at PPIP data from October 2013 until December 2016 on perinatal deaths due to IPA (unpublished), prolonged labour as an avoidable factor was identified in only $3 \%$ of cases.

- Most IPA deaths are caused by a lack of fetal monitoring. One could argue that a longer duration of labour creates more 'opportunity to lack monitoring'; however, this should not be a reason not to implement the updated guideline.

- Will fewer vaginal examinations lead to a delay in transfer?

- This has been accounted for in the guideline with 2-hourly examinations after $8 \mathrm{~cm}$.

- Assessment of the fetal condition and diagnosing cephalo-pelvic disproportion are vital for timely referrals.

- When implementing supportive care in labour, the right to privacy and the right to a companion in labour may cause 'conflicts' in some facilities.

- Full support of management is needed. Separating curtains can be an affordable way to create privacy and allow companions.

- Will spontaneous progress of labour lead to bed shortages?

- Midwife obstetric units (MOUs) may not have enough space to allow for longer latent phase. For this reason, the guideline recommends transfer from MOU to the next level of care after 12 hours.

- Less interventions (e.g. augmentation) could lead to less CDs (for fetal distress and/or poor progress) and a shorter admission: labour may take longer, but the woman can be discharged earlier after an uncomplicated vaginal delivery instead of $48-72$ hours after CD. The WHO recommends that all women should be observed in a facility for 24 hours after an uncomplicated vaginal delivery. However, due to bed shortages, women in SA can be discharged 6 hours after an uncomplicated vaginal delivery.

$\mathrm{OH}$ is still one the of the leading causes of maternal death worldwide and in SA. The majority of these deaths is potentially preventable and $30 \%$ is due to bleeding during/after CD. If unnecessary CDs could be avoided, the number of haemorrhage/CD related deaths could potentially be reduced.

A recent paper published by Solanki et al. ${ }^{[17]}$ reports on different modes of delivery in claims made to 10 different health insurance schemes in the private health sector in 2015: over 6500 births were analysed, resulting in a $\mathrm{CD}$ rate of $73.6 \%$. This shockingly high number was also recently reported in the media: for one medical scheme covering births in the private health sector in SA, $75 \%$ of all births happened via $\mathrm{CD}$. This number is more than double the $\mathrm{CD}$ rate of the world's richest countries (and American celebrity hospitals). ${ }^{[18]}$

The CLEVER package, as developed by Oosthuisen et al. ${ }^{[19]}$ demonstrates a solution to implementing respectful and high-quality maternity care. CLEVER is an acronym for Clinical care, Labour ward management, Eliminate barriers, Verify care, EOST (Emergency Obstetric Simulation Training) and Respectful care. A pilot study was done on the implementation in MOUs in Tshwane district. Data was collected through questionnaires in women who gave birth in 10 facilities. Results showed that even though $55.2 \%$ of women were very satisfied with the treatment they received in labour ward, only $47.4 \%$ admitted to being treated with respect and $55.9 \%$ said the midwives were nice during delivery. After implementation of the CLEVER package in 5 intervention MOUs, the results drastically improved: $73.7 \%$ of women were very satisfied, $75.6 \%$ reported being treated with respect and $92 \%$ said the midwives were nice. Furthermore, after implementation, there was a significant decrease in perinatal mortality in the intervention MOUs: decrease in in-facility fresh stillbirths from 8.50 to 1.30 per 1000 births, decrease in meconium aspiration from 12.18 to 4.55 per 1000 live births and decrease in birth asphyxia from 15.56 to 8.12 per 1000 live births.

\section{The future}

Between 2015 and 2017, the perinatal mortality rate (PNMR) for infants $\geq 1000 \mathrm{~g}$ was $22.6 / 1000$ births, the early neonatal mortality rate (ENNR) was 6.6/1 000 live births and the stillbirth rate was $16.1 / 1000$ births. $^{[3,45]}$ The majority of antenatal stillbirths $(72.6 \%)$ were of low birthweight (LBW), the most common causes were unexplained stillbirth, HDP and APH (mostly abruptio placentae). Of all LBW antenatal deaths, $46 \%$ were unexplained. Other studies have demonstrated that there is an excess of small for gestational age babies in the unexplained group and the majority occur between 34 and 37 weeks' gestation. ${ }^{[9,20]}$ This suggests fetal growth restriction (FGR) is a major cause of these unexplained antenatal deaths, and this is not being detected antenatally. It is well known that routine clinical methods to detect poor fetal growth (palpation or symphysis fundal growth) are ineffective. ${ }^{[21]}$ Recently a two-stage routine conventional ultrasound in LIC was shown to have no effect on perinatal or maternal death or on antenatal attendance. ${ }^{[22]}$ Routine fetal movement counting has also been shown to be ineffective and cannot be used as a method to detect at-risk fetuses. ${ }^{[23]}$

Doppler ultrasound of the umbilical artery (UA) (a test to assess placental function thereby detect FGR) has been shown to reduce the PNMR by $29 \%$ when used in managing high-risk pregnancies. ${ }^{[2]}$ However, a systematic review by Goffinet et al. ${ }^{[25]}$ in 1997 and a further paper by Alfirevic et al. ${ }^{[26]}$ concluded that routine use of umbilical Doppler cannot be recommended. Although these recommendations were based on low-risk populations from highincome countries (HIC) they suppressed further research into continuous wave (CW) Doppler as a way of detecting fetuses at risk of stillbirth.

Umbiflow is a novel, locally developed continuous CW Doppler ultrasound device that measures the Resistance Index (RI) of the UA and plots this against the gestational age. Recently a study in a low income setting in SA found a 1.5\% prevalence of absent end diastolic flow (AEDF) in a population classified as having low risk pregnancies that was screened using Umbiflow. ${ }^{[27]}$ This is 10 -times the prevalence previously recorded in low-risk pregnancies in HICs. ${ }^{[28]}$ Use of this Doppler RI information reduced the macerated stillbirth rate by $60 \%$ and the overall PMNR by $42 \% \cdot{ }^{[27]}$ Abnormalities in the 
blood flow of the UA reflect placental disease and suggest that, using Umbiflow, undiagnosed placental insufficiency was being detected and acted upon, saving the life of the fetus. Further studies in 9 different sites around SA have demonstrated a prevalence of AEDF of $0.89 \%{ }^{[29]}$ In these 9 sites the PNMR of the fetuses that had an Umbiflow examination was 6.4/1 000 births, similar to that found in the first study. A study by Roussouw et al. ${ }^{[30]}$ has shown that introducing Umbiflow into the clinical system will be cost-effective if comprehensive emergency obstetric care facilities are available, and would be more cost-effective than other interventions such as introducing new vaccines, therapeutic feeding (for severe wasting) or antiretroviral treatment.

SA has the ability to deal with small neonates (28 weeks or $\geq 1000 \mathrm{~g}$ ) as evidenced by its low ENNR of 6.6/1 000 live births for babies weighing $\geq 1000 \mathrm{~g}$. Stillbirths, especially antenatal stillbirths, are the biggest challenge in curbing preventable perinatal deaths. The Umbiflow apparatus, developed by the SAMRC and CSIR, is inexpensive and low-level healthcare workers can be trained in its use within two weeks. ${ }^{[27]}$

Should Umbiflow be shown to be an effective screening test and the health system can manage the new women with fetuses identified as being at risk of stillbirth, the integration of Umbiflow into BANC Plus could significantly improve antenatal care in SA.

\section{Conclusion}

SA is well on the way to meeting the challenges preventing women from having a positive pregnancy and childbirth experience and may lead the way for other LMICs. Concentrating on improving antenatal and intrapartum care will go a long way in reducing mortality, but other aspects such as preventing deaths from obstetric haemorrhage must not be neglected.

\section{Acknowledgements. None.}

Author contributions. All authors contributed equally to this manuscipt.

Funding. None.

Conflicts of interest. None.

1. Wrld Health Organization, UNAIDS, UNFPA, UNICEF, UNWomen. The Global Strategy for Women's, Children's and Adolescents' Health (2016 - 2030). Survive, Thrive, Transform. 2018 report on progress towards 2030 targets. Geneva: WHO; 2018.

2. Statistics South Africa (StatsSA). Mortality and Causes of Death in South Africa, 2015. Findings from Death Notification Form. Pretoria: StatsSA, 2017.

3. Pattinson RC, Hlongwane TMAG, Gebhardt GS. 'Placental insufficiency' is under appreciated as a Pattinson RC, Hlongwane TMAG, Gebhardt GS. 'Placental insufficiency' is under appreciated as a
cause of perinatal mortality. Perinatal Problem Identification Program (PPIP), 2015 - 2017. National cause of perinatal mortality. Perinatal Problem Identification Program (PPIP), 2015 - 2017.

4. Pattinson RC, Rhoda N, for the PPIP group. Saving Babies 2012 - 2013. Ninth Report on Perinatal Care 4. Pattinson RC, Rhoda N, for the PPIP group. Saving
in South Africa. Pretoria: Tshepesa Press, 2014.
5. National Committee on Confidential Enquiry into Causes of Maternal Deaths (NCCECMD). Saving Mothers 2017. Annual report, update on caesarean delivery mortality, new management of Saving Mothers 2017. Annual report, update on caesarean delivery mortality, new management of
hypertensive diseases in pregnancy guidelines and monitoring and evaluation of Basic Antenatal Care Plus implementation in South Africa. Pretoria: NCCECMD, 2017.

6. World Health Organization. Recommendations on Antenatal Care for a Positive Pregnancy Experience. Geneva: WHO, 2016.

7. World Health Organization. Recommendations: Intrapartum Care for a Positive Childbirth Experience. Geneva: WHO, 2018.

8. World Health Organization (WHO). Recommendations on Antenatal Care Contact Schedule. Geneva: WHO, 2016.

9. Lavin T, Preen DB, Pattinson R. Timing and cause of perinatal mortality for small-for-gestational age babies in South Africa: Critical periods and challenges with detection. Maternal Health Neonatol Perinatol 2016;2:11. https://doi.ord/10.1186/s40748-016-0039-4

10. Hlongwane TMAG, Pattinson RC. Monitoring and Evaluation of Basic Antenatal Care Plus Implementation in South Africa. Priorities in Perinatal Care Conference, 12-15 March 2019. Hartenbos, Western Cape.

11. Moodley J, Soma-Pillay P, Buchmann E, Pattinson RC. Managing Hypertensive diseases in Pregnancy. S Afr Med J 2019;109(9)

12. Zhang J, Troendle J, Mikolajczyk R, Sundaram R, Beaver J, Fraser W. The natural history of the normal first stage of labor. Obstet Gynecol 2010;115(4):705-710. http://doi.org/10.1097/ the normal first stage

13. Zhang .0b013e3181d55925.
Landy HJ, Branch DW, et al. Contemporary patterns of spontaneous labor with normal neonatal Zhang , Landy HJ, Branch DW, et al. Contemporary patterns of spontaneouslabor with normal neon
outcomes. Obstet Gynecol 2010;116(6):1281-1287. https://doi.org/10.097/aog.0b013e3181fdef6e.

14. Oladapo OT, Diaz V, Bonet $\mathrm{M}$, et al. Cervical dilatation patterns of 'low-risk' women with spontaneous labour and normal perinatal outcomes: A systematic review. BJOG 2018;125(8):944-954. http://doi. org $/ 10.1111 / 471-0528.14930$

15. Oladapo OT, Joao Paulo Souza JP, Bukola Fawole B, et al. Progression of the first stage of spontaneous labour: A prospective cohort study in two sub-Saharan African countries. PLoS Med 2018;15(1):e1002492. http://doi.org/10.1371/journal.pmed

16. Souza JP, Oladapo OT, Fawole B, et al. Cervical dilatation over time is a poor predictor of severe adverse birth outcomes: A diagnostic accuracy study. BJOG 2018;125(8):991-1000. https://doi. org/10.111/471-0528.15205

17. Solanki G, Fawcus S, Daviaud E. A cross sectional analytic study of modes of delivery and caesarean section rates in a private health insured South African population. PLoS One 2019;14(6):e0219020. https://doi.org/10.1371/journal.pone.0219020

18. Wasserman $\mathrm{H}$. The $\mathrm{C}$-section rate among Discovery members is now up to three times higher than at American celebrity hospitals - here's why. Business Insider. 2019. https://www.businessinsider.co.za/ discovery-c-sections-natural-births-2019-4 (accessed 22 April 2019)

19. Oosthuisen SJ. Respectful treatment of women in midwife obstetric units: an interventional study to improve clinical care in Tshwane District, South Africa. Pretoria: University of Pretoria, 2018. https:// www.up.ac.za/centre-for-maternal-fetal-newborn-and-child-healthcare/article/2744384/clevermaternity-care-research-centre-for-maternal-fetal-newborn-child-health-care-strategies (accessed 30 April 2019).

20. Allanson ER, Muller M, Pattinson RC. Causes of perinatal mortality and associated maternal complications in a South African province: Challenges in predicting poor outcomes. BMC Pre Childbirth. 2015;15:37. https://doi.org/10.1186/s12884-015-0472-9

21. Neilson JP. Symphysis fundal height measurement in pregnancy. Cochrane Database Syst Rev 2000; (2):CD000944.

22. Goldenberg RL, Nathan RO, Swanson D, et al. Routine antenatal ultrasound in low- and middleincome countries: First look- a cluster randomised trial. BJOG 2018;125(12):1591-1599. https://doi. org/10.1111/1471-0528.15287

23. Norman JE, Heazell AEP, Rodriguez A, et al. Awareness of fetal movements and care package to reduce fetal mortality (AFFIRM): A stepped wedge, clutster-randomised trial. Lancet 2018; 392(10158):1629-1638. 24. Alfirevic Z, Stampalija T, Gyte GML. Fetal and umbilical Doppler ultrasound in a high risk pregnancy. Cochrane Database Syst Rev 2013;11:CD 007529. https://doi.org/10.1002/14651858

25. Goffinet F, Paris J, Heim N, Nisand I, Breart G. Predictive value of Doppler umbilical artery velocimetry in low risk population with normal fetal biometry. A prospective study of 2016 women. Eur J Obste Gynecol Reprod Biol 1997;71(1):11-19.

26. Alfirevic Z, Stampalija T, Gyte GML. Fetal and umbilical Doppler ultrasound in normal pregnancy Cochrane Database Syst Rev 2010;4(8):CD001450. https://doi.org/10.1002/14651858.

27. Nkosi S, Makin J, Hlongwane TMAG, Pattinson RC. Screening and managing a low-risk pregnant population using continuous-wave Doppler ultrasound in a low-income population: A cohort analytical study. S Afr Med J 2019;109(5):347-352. https:///oi.org/10.7196/SAMJ.2019.v109i5.13611

28. Doppler French Study Group. A randomised controlled trial of Doppler ultrasound velocimetry of the Doppler French Study Group. A randomised controlled trial of Doppler ultrasound ve
umbilical artery in low risk pregnancies. Br J Obstet Gynaecol 1997;104:(4):419-424.

29. Hlongwane TMAG, Pattinson RC. The Prevalence of Abnormal Dopplers in South Africa. Priorities in Perinatal Care Conference. 12 - 15 March 2019. Hartenbos, Western Cape.

30. Rossouw L, Nkosi S, Pattinson RC. Cost-effectiveness of Umbiflow Doppler to screen and manage a low risk pregnant population in a middle-income country. Priorities in Perinatal Care Conference. 7 - 10 March 2017. Mpekweni Beach Resort, Eastern Cape. 